\title{
Supporting Information: Excitonic Dynamics in Janus MoSSe and WSSe Monolayers
}

Ting Zheng, ${ }^{\dagger} \ddagger, \perp$ Yu-Chuan Lin, ${ }^{*}, \boldsymbol{\Phi}, \S, \perp$ Yiling Yu, ${ }^{\boldsymbol{9}}$ Pavel Valencia-Acuna, ${ }^{\ddagger}$

Alexander A. Puretzky, "Riccardo Torsi, $§$ Chenze Liu, "llia N. Ivanov, " Gerd Duscher," David B. Geohegan, "Zhenhua Ni, ${ }^{* \dagger}$ Kai Xiao, " and Hui Zhao,

$\dagger$ School of Physics and Key Laboratory of MEMS of the Ministry of Education, Southeast University, Nanjing 211189, China

$\ddagger$ Department of Physics and Astronomy, The University of Kansas, Lawrence, Kansas 66045, United States

\Center for Nanophase Materials Sciences, Oak Ridge National Laboratory, Oak Ridge, Tennessee 37831, United States

$\S$ Department of Materials Science and Engineering, The Pennsylvania State University, University Park, Pennsylvania 16802, United States

||Department of Materials Science and Engineering, University of Tennessee, Knoxville, Tennessee 37996, United States $\perp$ Contributed equally to this work

E-mail: yul194@psu.edu; zhni@seu.edu.cn; huizhao@ku.edu 


\section{Experimental Methods}

CVD synthesis of $\mathbf{W S}_{2}$ and $\mathrm{MoS}_{2}$ monolayers. The synthesis of $\mathrm{WS}_{2}$ monolayers was performed using sulfur powder (Sigma-Aldrich) that was placed $20 \mathrm{~cm}$ upstream from the center heat zone where $\mathrm{WO}_{3}(10 \mathrm{mg}, 99.99 \%$, Sigma-Aldrich) powder mixed with $3 \%$ of $\mathrm{KCl}$ powder (Sigma-Aldrich), by mass, was placed. $\mathrm{SiO}_{2} / \mathrm{Si}$ substrates also were placed in the center of the 2-inch tube furnace, facing down above mixed $\mathrm{WO}_{3}-\mathrm{KCl}$ powders by 2-3 mm. A typical growth run was performed at $820-850^{\circ} \mathrm{C}$ for 5 minutes under a flow of $\mathrm{Ar}$ gas at 60 standard cubic centimeters per minute (sccm) and 730 Torr. CVD MoS $\mathrm{S}_{2}$ monolayers were grown using a mixture of $\mathrm{MoO}_{3}(5 \mathrm{mg})$ and $\mathrm{S}$ powders at $750^{\circ} \mathrm{C}$ for $4-6$ minutes under a flow of Ar gas at $70 \mathrm{sccm}$ and 730 Torr.

Pulsed laser deposition of Se for Janus monolayers. A pulsed $\mathrm{KrF}$ (248 nm, $25 \mathrm{~ns}$ FWHM, 1-5 Hz repetition rate) laser was used for the ablation of the targets in Ar background gas. A 1-inch-diameter selenium pellet (Plasmaterials, Inc., 99.99\% purity) was used as the ablation target. Using a projection beamline, an aperture was imaged onto the target to produce a $1.25 \mathrm{~mm} \times 4.5 \mathrm{~mm}$ rectangular spot. Typically, $57 \mathrm{~mJ}$ of energy was used, providing $1.0 \mathrm{~J} \mathrm{~cm}^{-2}$ per laser pulse at the target surface. $\mathrm{WS}_{2}$ and $\mathrm{MoS}_{2}$ monolayers grown by CVD on $\mathrm{SiO}_{2} / \mathrm{Si}$ substrates were mounted on a 1-inch-diameter heater (Heat Wave Laboratories, Inc.) placed $10 \mathrm{~cm}$ away from the Se target in a cylindrical stainless-steel chamber $(50 \mathrm{~cm}$ inner diameter, $36 \mathrm{~cm}$ tall). The heater temperature was controlled to \pm $2^{\circ} \mathrm{C}$ via a PID controller, and ramp-rates were typically $30^{\circ} \mathrm{C}$ per minute. The gas pressure was controlled with a mass flow controller (Ar 99.995\%, $10 \mathrm{sccm}$ ) and a downstream throttle valve. The pressure was set at 20 mTorr, and the temperature was set at $300^{\circ} \mathrm{C}$ for achieving Janus monolayers. ${ }^{1}$

Absorbance spectroscopy. The absorbance spectra were measured using a laser-driven white light source (EQ-99FC, Energetiq). The white light source was coupled out to a $25 \mu \mathrm{m}$ diameter optical fiber and was focused on the sample surface to a $1.5 \mu \mathrm{m}$ spot using two microscope objectives: a $5 \times$ collimating objective, NA (numeric aperture) $=0.1$ 
and a $100 \times$ long-working-distance objective, $\mathrm{NA}=0.8$, in an inverted microscope. The transmitted light was collected by a $50 \times$ long-working-distance objective $(\mathrm{NA}=0.5)$ in an upright microscope coupled to the inverted microscope and was analyzed by a spectrometer (Spectra Pro 2300i, Acton) equipped with a charge-coupled-device (CCD) camera (Pixis 256BR, Princeton Instruments). The absorbance $(A)$ was calculated as $A=\log _{10}\left(I_{0} / I\right)$, where $I$ and $I_{0}$ are the light intensities transmitted through the substrate on and off a TMD crystal, respectively.

Photoluminescence spectroscopy. The PL spectroscopy was performed with a homemade setup with a 405-nm continuous-wave diode laser (Thorlabs) as the excitation source. With a $100 \times$ and NA $=0.75$ objective lens, the focused laser spot size was about $1 \mu \mathrm{m}$. The PL was collected by the same objective lens and was sent to a spectrometer (Horiba iHR550) equipped with a thermoelectrically cooled CCD camera (Synapse).

Differential reflectance spectroscopy. The differential reflectance measurements were performed with a homemade setup, as shown schematically in Figure S1. ${ }^{2}$ The setup is based on a femtosecond laser system that is composed of a Ti:sapphire oscillator, an optical parametric oscillator (OPO), a supercontinuum generator (SCG), and a second harmonic generator (SHG). The Ti:sapphire oscillator (Newport Spectra-Physics) produces 100fs pulses that is tunable in the range of $700-900 \mathrm{~nm}$ with a repetition rate of $80 \mathrm{MHz}$ and an average power of $2 \mathrm{~W}$. Part of the Ti:sapphire output was guided into the SCG, which is composed of a photonic crystal fiber and other optical components (Newport) and produces a coherent and broadband irradiation. A bandpass filter (with a bandwidth of $10 \mathrm{~nm}$ ) is used to select the desired wavelength component. The OPO (Newport Spectra-Physics), pumped by the Ti:sapphire, produces near infrared pulses, which can be converted to visible pulses by SHG using a nonlinear optical crystal. The output of the Ti:sapphire oscillator can also produce its second harmonic directly by the SHG unit. For a certain measurement, two of these pulses were selected as the pump and probe. It should be noted that although the system is capable of covering a wavelength range from 350 - $2350 \mathrm{~nm}$, there are limitations 


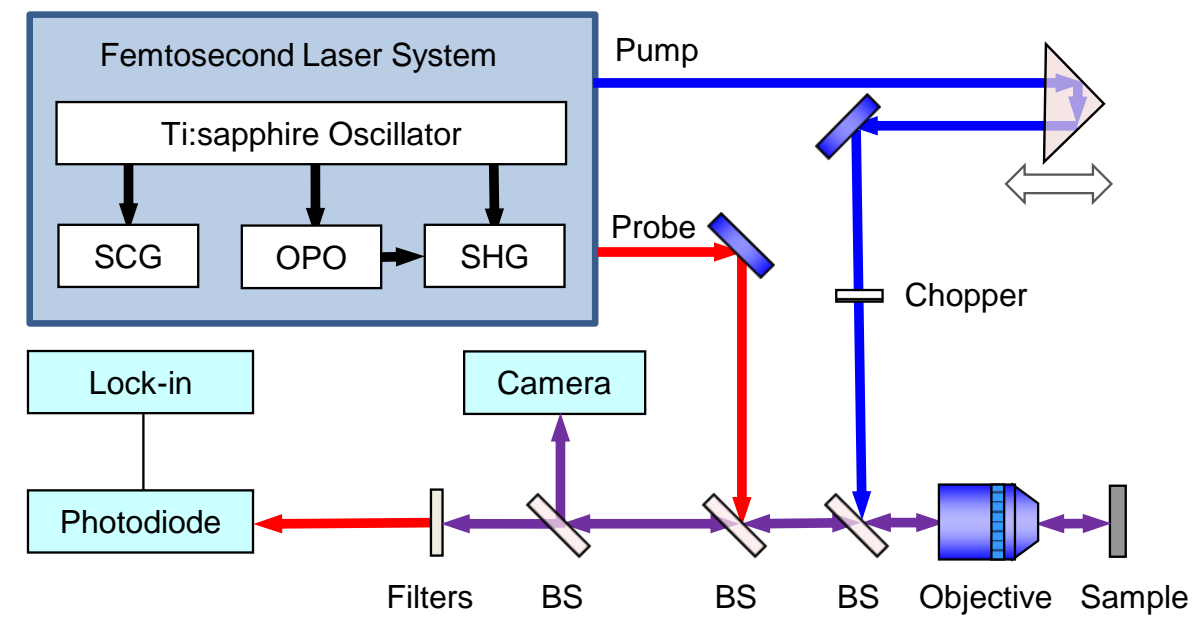

Figure S1: The differential reflectance spectroscopy setup.

when multiple wavelengths are needed because of the dependence of the SCG and OPO on the Ti:sapphire wavelength. The pump and probe beams are combined by a beamsplitter (BS) and are co-focused to the sample to spot sizes of about $1 \mu \mathrm{m}$ by a microscope objective lens $(\mathrm{NA}=0.75)$. Before entering the $\mathrm{BS}$, the pump beam is sent to a retroreflector that is mounted on a motorized linear stage, so that its propagation distance, and hence its arrival time at the sample with respect to the probe pulse, is controlled. A mechanical chopper is used to modulate the pump intensity entering the sample at about $3 \mathrm{kHz}$. The reflected probe and pump from the sample are collimated by the the objective lens. A camera is used to monitor their focused spots on the sample as well as the sample surface during the alignment process. A filter is used to block the unwanted pump reflection, allowing only the probe to reach a silicon photodiode. The output voltage of the photodiode is measured by a lock-in amplifier at the chopping frequency. With this setup, we can measure the differential reflectance of the probe, $\Delta R / R_{0}=\left(R-R_{0}\right) / R_{0}$, where $R$ and $R_{0}$ are the reflectance of the sample with and without the presence of the pump beam. 


\section{Structural Characterization of Janus MoSSe and WSSe Samples}

High-angle annular dark field scanning transmission electron microscope (HAADF-STEM) images of $\mathrm{WS}_{2}$ and WSSe monolayers [Figure S2(a)-S2(b)] and those of $\mathrm{MoS}_{2}$ and MoSSe [Figure S2(c)-S2(d)] along the (001) direction show the structures remain in 2H-phase after the Se implantation. In Figure S2(a), the Z-contrast intensity profile extracted along the colored box in $\mathrm{WS}_{2}$ shows the intensity ratio $I_{W} / I_{2 S}$ of 3.17. In Figure $\mathrm{S} 2(\mathrm{~b})$, the chalcogenide columns are seen to become brighter after the topmost $\mathrm{S}$ were replaced with Se. The intensity ratio $I_{W} / I_{S+S e}$ was reduced to 1.67 in the line profile. The intensity profiles along the colored boxes in Figure $\mathrm{S} 2(\mathrm{c})-\mathrm{S} 2(\mathrm{~d})$ indicate the ratio $I_{M o} / I_{2 S}$ of 3.5 was reduced to the ratio of $I_{M o} / I_{S+S e}$ of 1.13 . The intensity ratios for both WSSe and MoSSe agree well with the theoretical intensity ratios that utilize the $\mathrm{Z}^{x}$ contrast dependency for each element, where $x$ is the scaling power typically between $1.6-2.0 .^{1,3}$

(a)

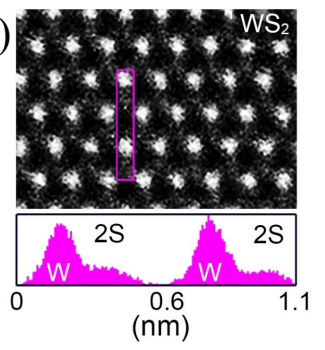

(c)

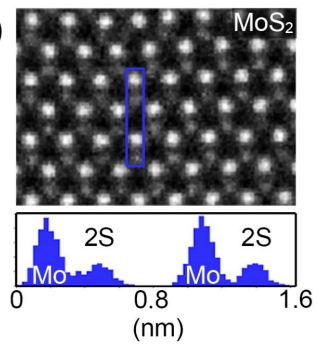

(b)

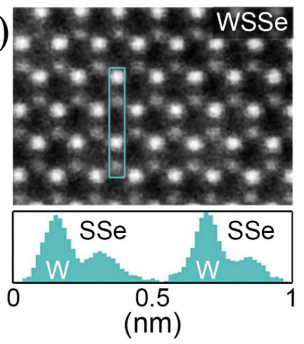

(d)

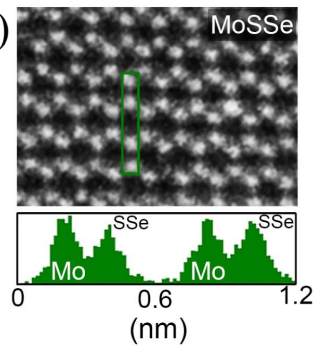

(e)
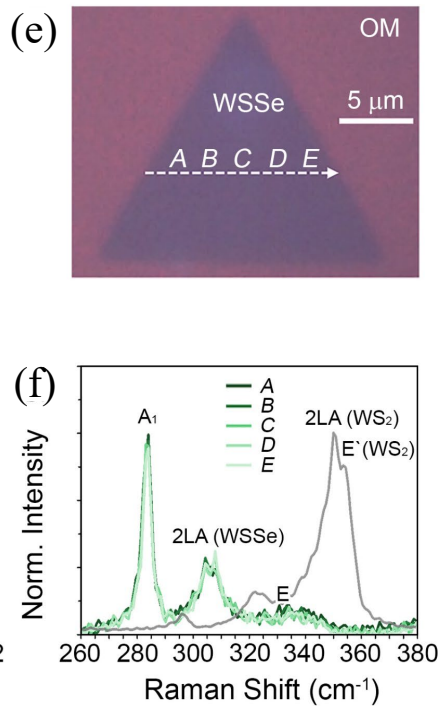

$(\mathrm{g})$

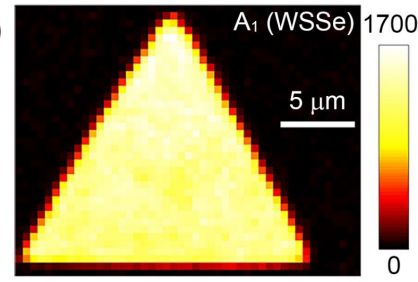

(h)

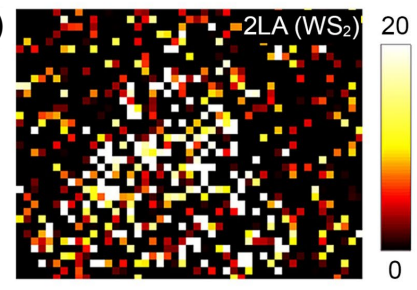

Figure S2: (a)-(b) HAADF images of $\mathrm{WS}_{2}$ and Janus WSSe monolayers. The selected line proles in the images show z-contrast between $\mathrm{W}$ and chalcogen sites. (c)-(d) HAADF images and intensity line proles for $\mathrm{MoS}_{2}$ and MoSSe. (e) An optical microscope (OM) image of the Janus WSSe monolayer. (f) Raman spectra (under 532-nm excitation) obtained from the positions labeled in (e). The gray solid line is the Raman spectrum of a $\mathrm{WS}_{2}$ monolayer for comparison. (g)-(h) The intensity maps for the Raman peaks of the domain shown in (e) at $284 \mathrm{~cm}^{-1}\left(\mathrm{~A}_{1}, \mathrm{WSSe}\right)$ and $352 \mathrm{~cm}^{-1}\left(2 \mathrm{LA}, \mathrm{WS}_{2}\right)$, respectively. The comparison between the intensities of these two maps confirms the $\mathrm{WS}_{2}$ has been fully converted to WSSe. 
The quality and the uniformity of the Janus samples were further confirmed by Raman intensity mapping of a WSSe sample shown in Figure S2(e), which was converted from a $\mathrm{WS}_{2}$ flake. The intensity of one of the $A_{1}$ modes $\left(284 \mathrm{~cm}^{-1}\right)$ is uniform across the domain, as shown in Figure $\mathrm{S} 2(\mathrm{~g})$, while the originally intense $2 L A$ mode of $\mathrm{WS}_{2}\left(352 \mathrm{~cm}^{-1}\right)$ is completely diminished [Figure S2(h)]. The Raman spectra from the 5 points indicated in Figure S2(e) are consistent, as shown in Figure S2(f).

The spectroscopic and dynamical properties of excitons discussed in the main text also provide important, albeit indirect, information on the crystalline quality and lattice strain in Janus TMDs. Because Janus structures show broader PL linewidth, one may wonder how the S-to-Se conversion affects the material properties. Our previous investigation that characterized the Janus monolayers with X-ray photoelectron spectroscopy (XPS) and advanced
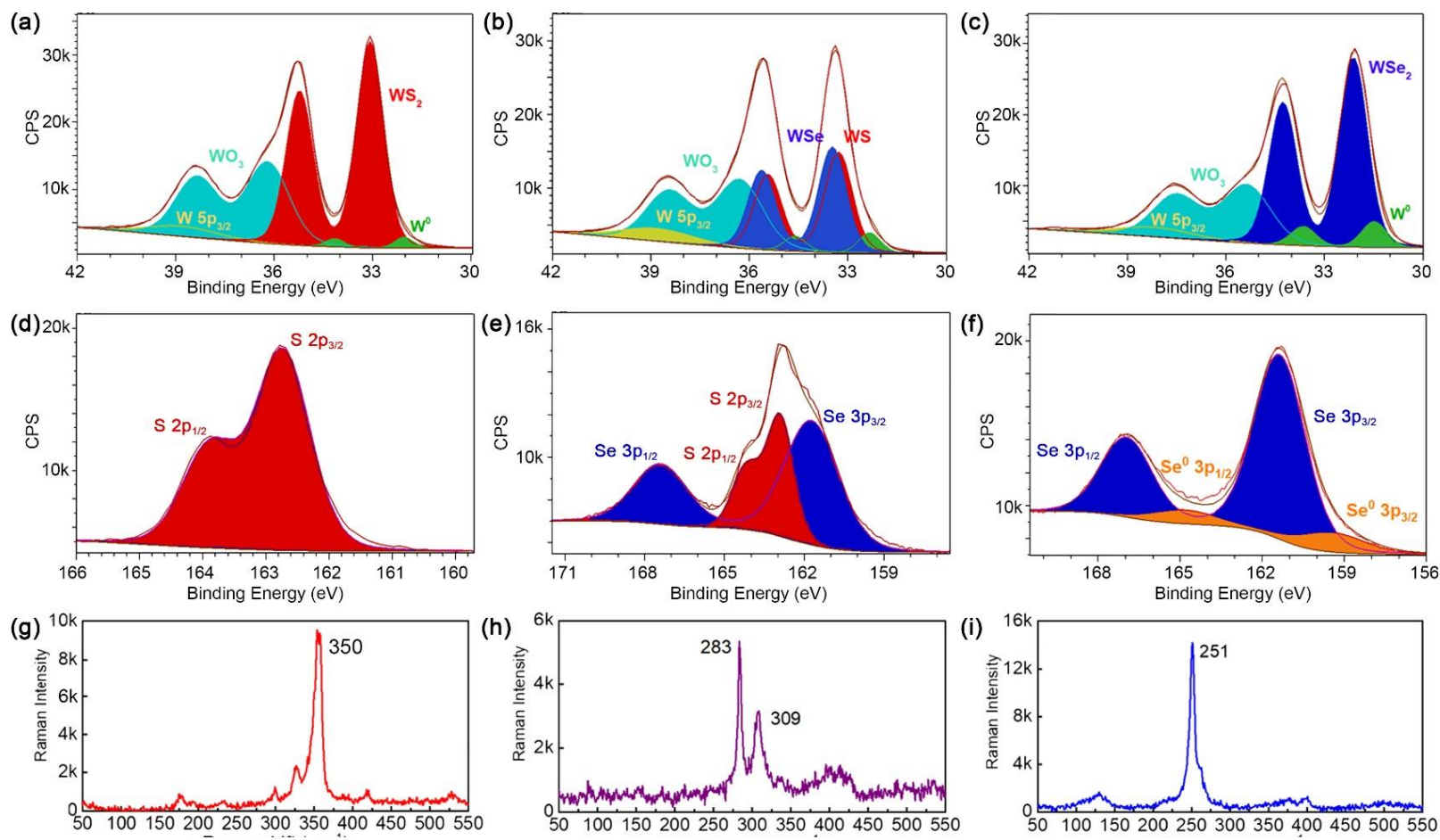

Figure S3: (a-c) W-4f binding energy of as-received $\mathrm{WS}_{2}(\mathrm{a})$, converted Janus WSSe (b), and converted $\mathrm{WSe}_{2}$ (c) on sapphire. The black and red outer curves are original and fitted spectra, respectively. (d-e) S-2p and Se-3p binding energy of as-received $\mathrm{WS}_{2}$ (d), converted Janus WSSe (e), and converted $\mathrm{WSe}_{2}$ (f) on sapphire. (g-i) The Raman spectra of as-received $\mathrm{WS}_{2}(\mathrm{~g})$, converted Janus WSSe (h), and converted $\mathrm{WSe}_{2}(\mathrm{i})$ on sapphire. 
Table S1: Summary of the fitted components of $\mathrm{W}-4 \mathrm{f}$ for as-received $\mathrm{WS}_{2}$, converted Janus WSSe, and converted $\mathrm{WSe}_{2}$.

\begin{tabular}{c|c|c|c}
\hline \hline Component & $\mathrm{WS}_{2}$ & Janus WSSe & $\mathrm{WSe}_{2}$ \\
\hline $\mathrm{W} 4 \mathrm{f}(\mathrm{W}-\mathrm{S})$ & 0.5840 & 0.2842 & 0 \\
$\mathrm{~W} 4 \mathrm{f}(\mathrm{W}-\mathrm{Se})$ & 0 & 0.2984 & 0.6069 \\
$\mathrm{WO}_{3}$ & 0.3644 & 0.3185 & 0.3204 \\
$\mathrm{~W}^{0}$ & 0.0208 & 0.0441 & 0.0667 \\
$\mathrm{~W} 5 \mathrm{p}_{3 / 2}$ & 0.0424 & 0.0545 & 0.0424 \\
\hline
\end{tabular}

HAADF-STEM already confirms that the Se implantation does not degrade the crystalline quality but replacing the top $\mathrm{S}$ atoms to form a Janus structure. ${ }^{1}$

Here, we discuss the compositional change in $\mathrm{WS}_{2}$ monolayer after Se implantation. We fitted the W, S, and Se binding energies of our $\mathrm{WS}_{2}$, WSSe, and $\mathrm{WSe}_{2}$ samples. The continuous $\mathrm{WS}_{2}$ monolayer film on a $1 \mathrm{~cm}^{2}$ sapphire that we used in the XPS investigation was purchased from a vendor (2D Semiconductors). Because of its uniform monolayer coverage over $1 \mathrm{~cm}^{2}$ area, this type of sample is desired for our XPS measurements that use a $400-\mu \mathrm{m}$ beam size. The same $\mathrm{WS}_{2} /$ sapphire was split into a few smaller pieces for the selenization that converts $\mathrm{WS}_{2}$ into $\mathrm{WSSe}$ and $\mathrm{WSe}_{2}$. The fitted binding energies of as-received $\mathrm{WS}_{2}$, converted Janus WSSe, and converted $\mathrm{WSe}_{2}$ are shown in Figure S3(a)-S3(c)]. The fractions of the fitted components of these samples are summarized in Table S1.

These results indicate that $\mathrm{WS}_{2}$ on sapphire from the vendor already had a significant amount of $\mathrm{WO}_{3}$ and metallic tungsten $\left(\mathrm{W}^{0}\right)$ prior to the selenization. The original fraction of $\mathrm{WO}_{3}$ in $\mathrm{WS}_{2}(0.3644)$ did not reduce significantly after $\mathrm{WS}_{2}$ was converted to Janus WSSe (0.3185) and $\mathrm{WSe}_{2}$ (0.3204). Additionally, the fractions of metallic $\mathrm{W}^{0}$ increased slightly after selenization due to the possible reduction of $\mathrm{WO}_{3}$. Because $\mathrm{WO}_{3}$ is likely independent of the selenizing processes for WSSe and $\mathrm{WSe}_{2}$, we conclude that $\mathrm{WO}_{3}$ is either 3D particle residue on the surface or intercalated in between the monolayer and sapphire. It is worth noting that the intercalation of molecules between as-grown monolayers and sapphire's surface was reported. ${ }^{4}$ In either case, the majority of $\mathrm{WO}_{3}$ was not affected by low-energy Se implantation. The fitted $\mathrm{W}-4 \mathrm{f}_{7 / 2}$ and $\mathrm{W}-4 \mathrm{f}_{5 / 2}$ doublets of $\mathrm{WS}_{2}$, WSSe, and 


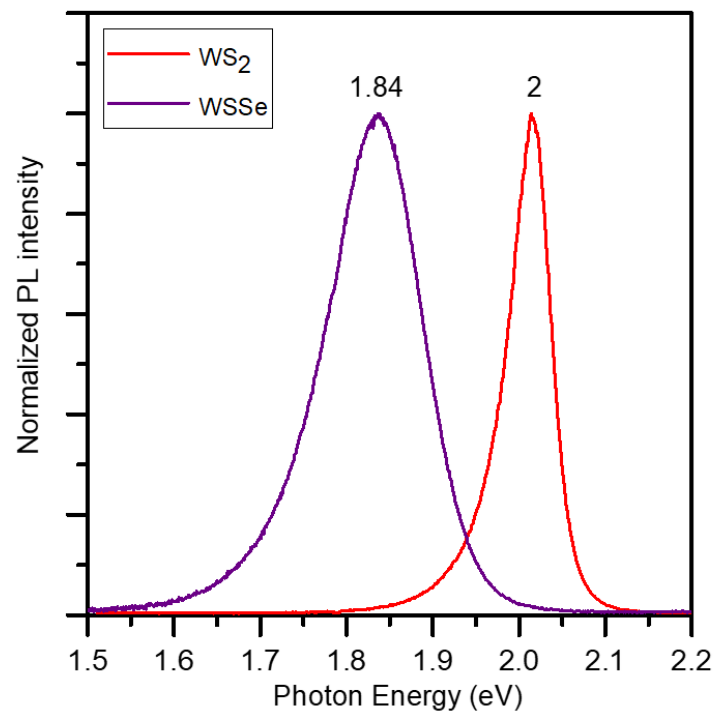

Figure S4: Normalized PL spectra (under 532-nm excitation) of the as-received $\mathrm{WS}_{2}$ mono-layer on sapphire (from the vendor 2D Semiconductors) and the Janus WSSe monolayer that is converted from it.

$\mathrm{WSe}_{2}$ show a clear transition from a single pair of doublets of W-S bond, to two pairs of doublets contributed by even amounts of W-S and W-Se bond, and to a pair of doublets of W-Se bonds. The ratio of W-Se/W-S based on their W $4 \mathrm{f}$ doublets in Janus WSSe [Figure $\mathrm{S} 3(\mathrm{~b})$ ] is about 1.05. The fitted S-2p/Se-3p doublets [Figure S3(d)-S3(f)] also show the same transition from $\mathrm{WS}_{2}$ to $\mathrm{WSSe}$ and $\mathrm{WSe}_{2}$. The Se/S ratio of Janus WSSe from the fitting of S-2p/Se-3p [Figure S3(e)] is consistently 1.05. The Raman spectra of as-received $\mathrm{WS}_{2}$ and selenized samples [Figure S3(g)-S3(i)] show the characteristic peaks of $\mathrm{WS}_{2}\left(350 \mathrm{~cm}^{-1}\right)$, Janus WSSe $\left(283 \mathrm{~cm}^{-1}\right.$ and $\left.309 \mathrm{~cm}^{-1}\right)$, and WSe2 $\left(251 \mathrm{~cm}^{-1}\right)$. Finally, the PL peak position of the monolayer [Figure S4] shifts from $2 \mathrm{eV}\left(\mathrm{WS}_{2}\right)$ to $1.84 \mathrm{eV}$ (Janus WSSe). Therefore, we conclude that the phonon structures and optical properties of converted Janus WSSe monolayers are not affected by the $\mathrm{WO}_{3}$ residue.

In addition, the emerged Se-3d core level doublets [Figure S5] are consistent with those of $\mathrm{WS}_{x} \mathrm{Se}_{y}$ alloys in the literature. ${ }^{5}$ There is no sign of the Se-O peak of selenium oxides at $59 \mathrm{eV}$, indicating the Janus WSSe is free of oxygen incorporation.

Next, we address the issue of lattice strain in Janus structures. Atomic force microscope 


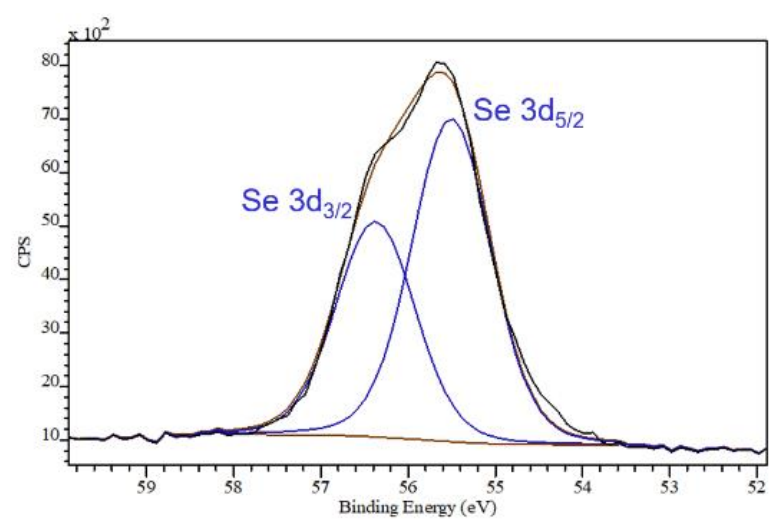

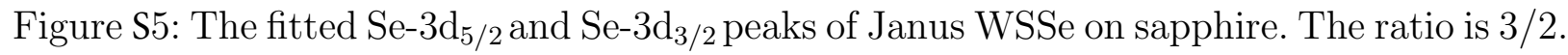

(AFM) images of $\mathrm{WS}_{2}$ and Janus WSSe provide a before-and-after comparison of their morphology [Figure $\mathrm{S} 6(\mathrm{a})-\mathrm{S} 6(\mathrm{~b})$ ]. The surface of $\mathrm{WS}_{2}$ on $\mathrm{SiO}_{2}$ was flat. After the conversion, the surface of Janus WSSe started to have 3-fold symmetry wrinkles $3-5 \mathrm{~nm}$ tall and $10-20$ $\mathrm{nm}$ wide on average. According to theory, a freestanding monolayer Janus TMD is expected to curve with a radius of $8 \mathrm{~nm}$ due to different lattice constants on the two sides. ${ }^{6,7}$
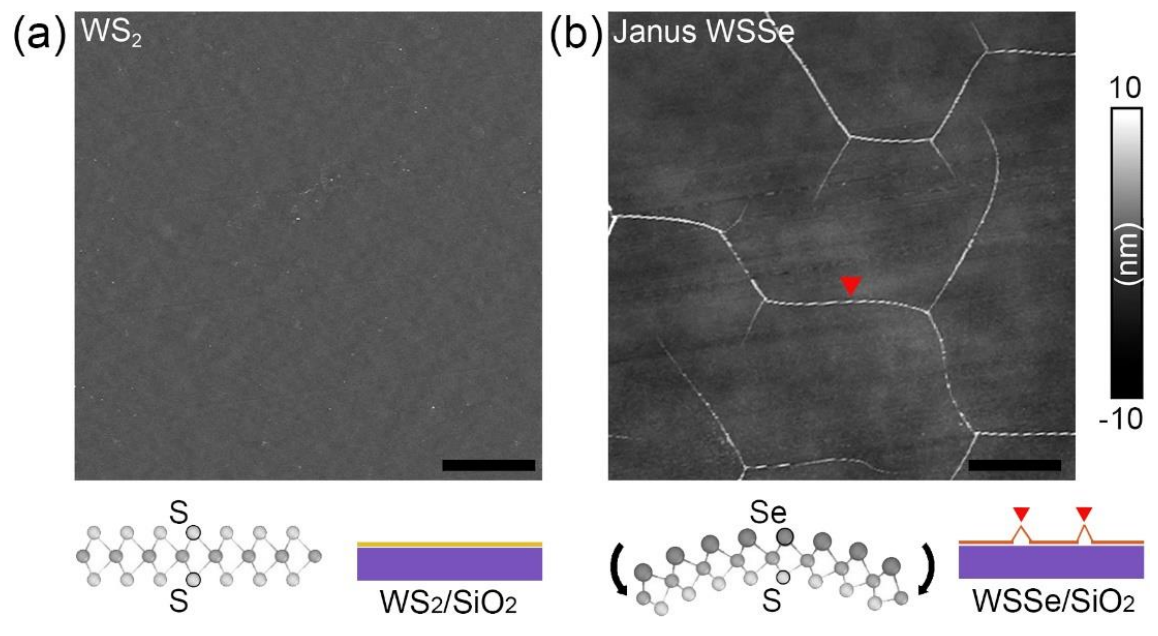

Figure S6: (a) An atomic force microscopic (AFM) image of CVD monolayer $\mathrm{WS}_{2}$, an atomic model of monolayer $\mathrm{WS}_{2}$, and a schematic of $\mathrm{WS}_{2} / \mathrm{SiO}_{2}$ present a flat surface before Se implantation. (d) After the $\mathrm{WS}_{2}$ is converted to Janus WSSe, wrinkles marked by a red triangle are seen in the AFM image. Unable to be freestanding as a natural curvature, Janus WSSe were kept flat on $\mathrm{SiO}_{2}$ but produced wrinkles to accommodate biaxial compressive strain. (Scale bar: $1 \mu \mathrm{m}$ ) 
However, their strong interactions with $\mathrm{SiO}_{2}$ keep them on the surface. ${ }^{8}$ Therefore, such wrinkles form in Janus TMD due to the substrate constraint and the compressive strain. These limitations could be responsible for the broader PL linewidth and shorter exciton nonradiative recombination lifetime observed on Janus WSSe, compared to pristine $\mathrm{WS}_{2}$.

\section{Janus WSSe Power Dependence}

Photoluminescence spectra of the Janus WSSe sample under different excitation powers were measured, as shown in Figure S7. The spectral shape shows no changes, while the PL intensity is proportional to the excitation power.
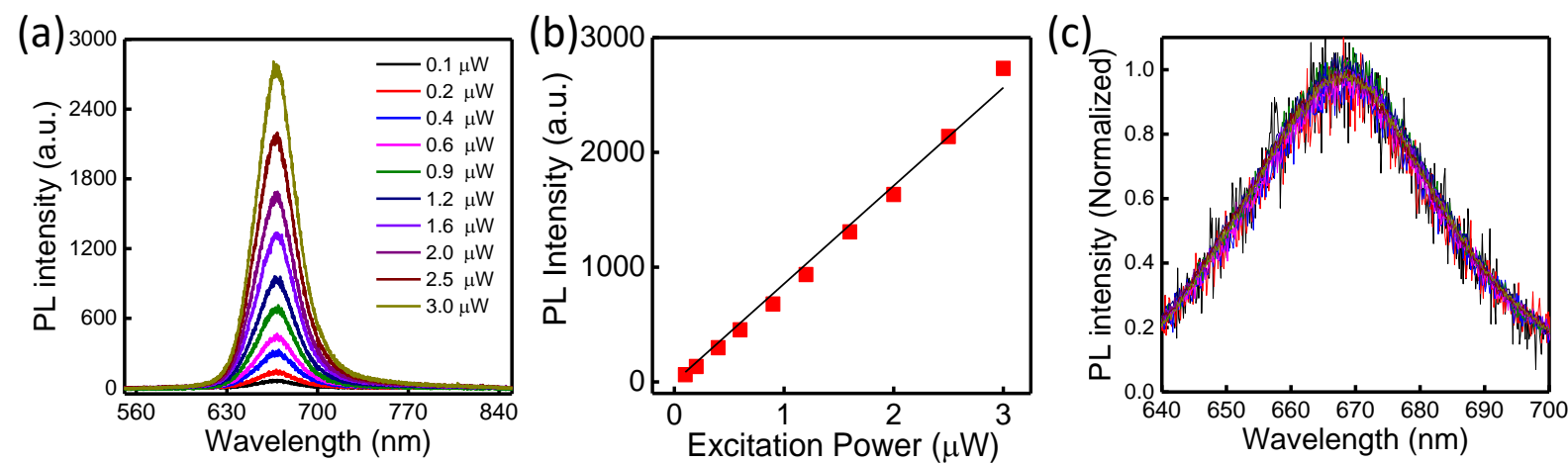

Figure S7: (a) PL spectra of Janus WSSe under different excitation powers. (b) Peak PL intensity of Janus WSSe as a function of the excitation power. (c) Normalized spectra from (a) to show that the spectral shape is independent of the excitation power.

The differential reflectance measurement of Janus WSSe was repeated with various pump fluences. The peak signal was found to be proportional to the pump fluence and the shape of the decay curves remained the same, as shown in Figure S8.

\section{Second-Harmonic Generation Measurements}

Second-harmonic generation (SHG) measurements were performed on Janus WSSe in comparison with $\mathrm{WS}_{2}$ monolayer. Figure S9(a) shows a sample that contains monolayer and bilayer regions of $\mathrm{WS}_{2}$. We first performed $\mathrm{SHG}$ spectroscopy on the monolayer region, with an 800-nm pulse incident normal to the sample. The polarization of the incident pulse was 


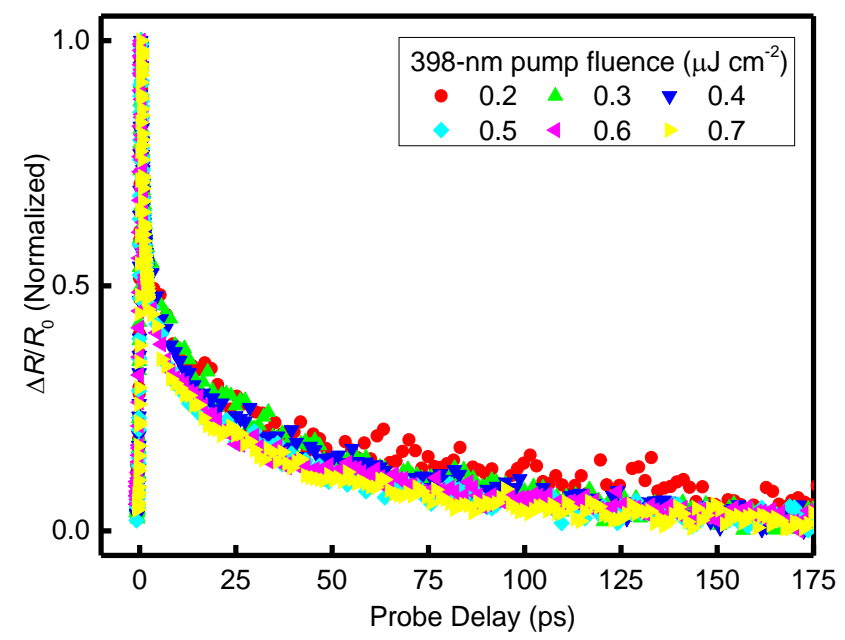

Figure S8: Normalized differential reflectance signal from Janus WSSe with a probe photon energy of $1.893 \mathrm{eV}$ measured with different values of pump fluence as labeled, illustrating the independence of the decay dynamics of the signal on the pump fluence.

perpendicular to one edge of the flake, as indicated by the arrow in Figure S9(a). This is the armchair direction with the expected maximal SHG. ${ }^{9}$ The black curve in Figure S9(b) shows the obtained SH spectrum. When the laser spot was moved to the bilayer region, the SH intensity is decreased by about two orders of magnitude, as shown by the black curve in Figure S9(c). The weak SHG of TMD bilayers is well known, due to the inversion symmetry of their lattice structure. ${ }^{9-11}$

(a)

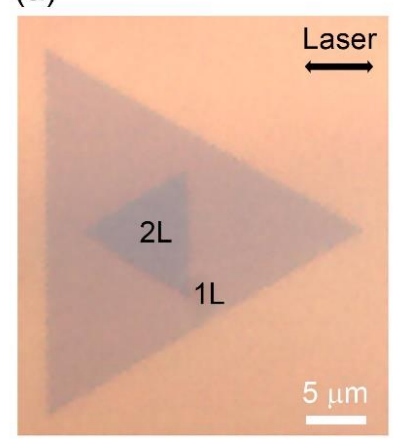

(b)

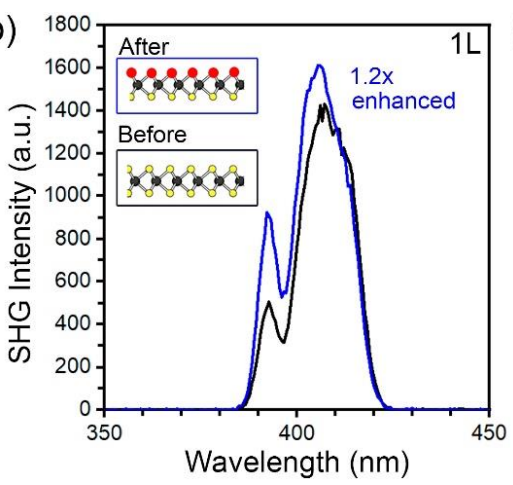

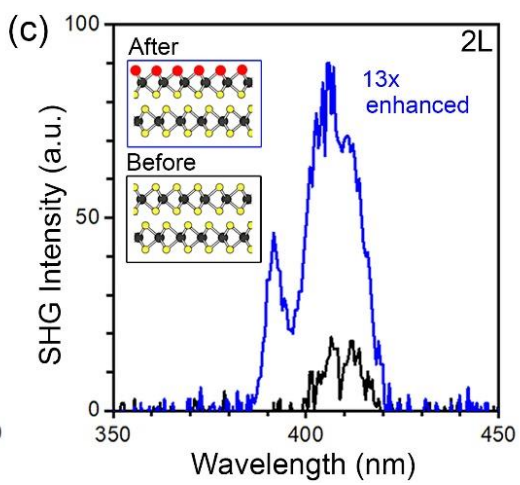

Figure S9: (a) The optical microscope image of a $\mathrm{WS}_{2}$ sample contains both monolayer $(1 \mathrm{~L})$ and bilayer $(2 \mathrm{~L})$ regions. (b) The second harmonic spectra of the monolayer region of the sample before (black) and after (blue) the Se-implantation. The inset shows the schematically the lattice structrues before (black box) and after (blue box) the conversion. (c) Same as (b) for the bilayer region. 
After these measurements, the sample was converted to the Janus structure by replacing the top S atoms with Se by implantation, as schematically illustrated by the insets of Figure S9(b) and S9(c). The SH spectrum of the monolayer WSSe shows minimal change after the Se implantation, as shown by the blue curve in Figure S9(b). Therefore, the conversion to the Janus structure does not significantly alter the SHG property of TMD monolayers for light polarized in-plane. This is expected, since the built-in dipole moment is perpendicular to the light polarization and thus does not enable the field-induced SHG, as for oblique incidence. ${ }^{12}$ However, as shown in Figure S9(c), the SH intensity of the bilayer region was increased by about 13 times. This shows that the conversion to the Janus structure broke the inversion symmetry and thus enabled the second-order nonlinear optical responses.

\section{Fast decay of differential reflectance due to exciton formation}

(a)

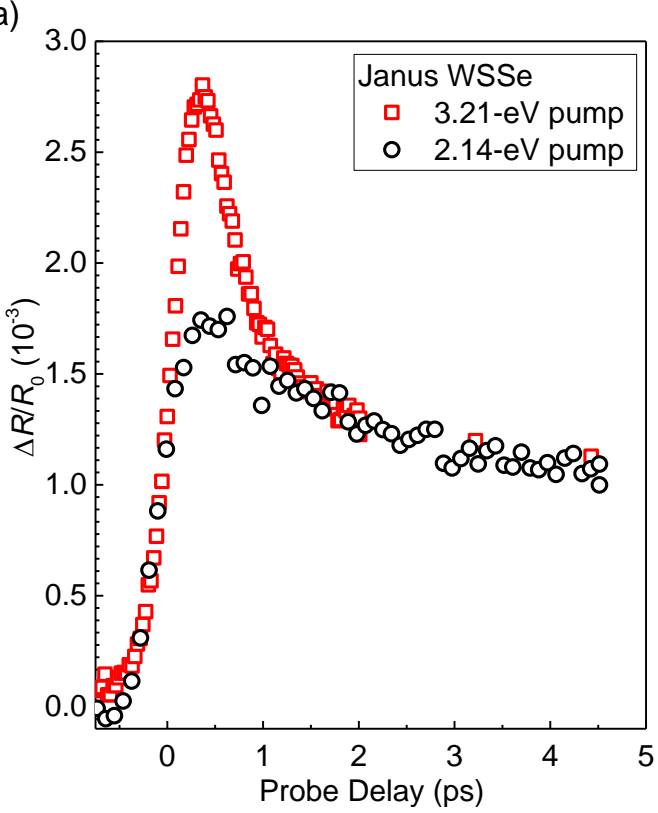

(b)

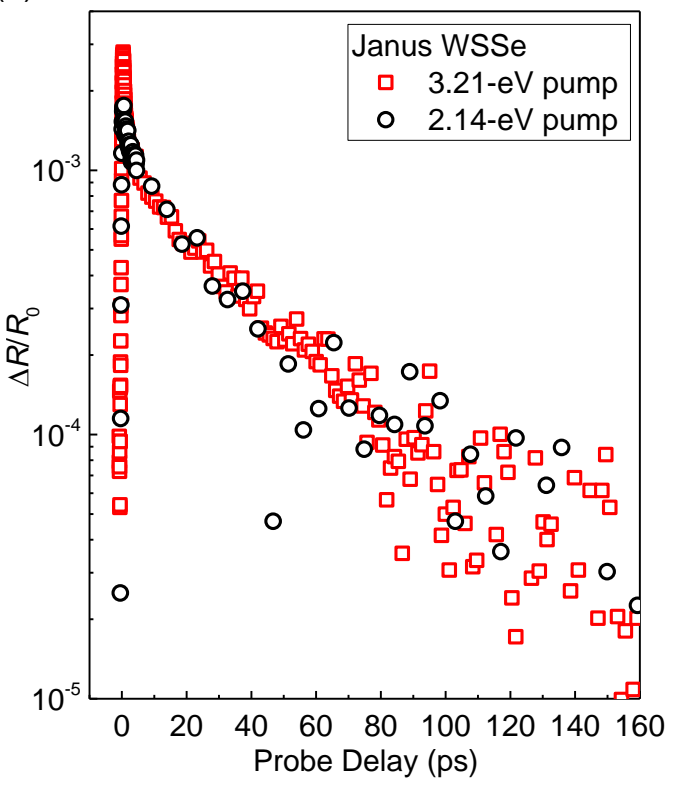

Figure S10: (a) The differential reflectance signal as a function of the probe delay from the Janus WSSe sample with the pump photon energies of $3.21 \mathrm{eV}$ (red squares) and $2.14 \mathrm{eV}$ (black circles), respectively. The latter is normalized to match the former for probe delays after 2 ps. (b) Same as (a) but is over a longer time range and plotted in semi-logarithmic scale.

To further confirm the assignment of the fast-decay component of the differential re- 
flectance signal to the exciton formation, we repeated the measurement on Janus WSSe with a $2.14 \mathrm{eV}$ pump, under otherwise the same conditions. Compared to the 3.21-eV pump, this pump is closer to the band edge and thus predominately injects excitons resonantly. As shown in Figure S10(a), the fast-decay component of the differential reflectance signal is largely absent, while the rest of the dynamics [Figure S10(b)] is similar to that observed with the 3.21-eV pump. This observation is consistent with previous results on pristine TMDs. ${ }^{13}$

\section{References}

1. Lin, Y.-C.; Liu, C.; Yu, Y.; Zarkadoula, E.; Yoon, M.; Puretzky, A. A.; Liang, L.; Kong, X.; Gu, Y.; Strasser, A.; Meyer, H. M.; Lorenz, M.; Chisholm, M. F.; Ivanov, I. N.; Rouleau, C. M.; Duscher, G.; Xiao, K.; Geohegan, D. B. Low Energy Implantation into Transition Metal Dichalcogenide Monolayers to Form Janus Structures. ACS Nano 2020, 14,3896 .

2. Ceballos, F.; Zhao, H. Ultrafast Laser Spectroscopy of Two-Dimensional Materials beyond Graphene. Adv. Funct. Mater. 2017, 27, 1604509.

3. Bangert, U.; Stewart, A.; O’Connell, E.; Courtney, E.; Ramasse, Q.; Kepaptsoglou, D.; Hofsäss, H.; Amani, J.; Tu, J.-S.; Kardynal, B. Ion-beam modification of 2-D materials single implant atom analysis via annular dark-field electron microscopy. Ultramicroscopy 2017, 176, $31-36$.

4. Zheng, C. X.; Xu, Z. Q.; Zhang, Q. H.; Edmonds, M. T.; Watanabe, K.; Taniguchi, T.; Bao, Q. L.; Fuhrer, M. S. Profound Effect of Substrate Hydroxylation and Hydration on Electronic and Optical Properties of Monolayer $\mathrm{MoS}_{2}$. Nano Lett. 2015, 15, 3096-3102.

5. Fu, Q.; Yang, L.; Wang, W. H.; Han, A.; Huang, J.; Du, P. W.; Fan, Z. Y.; Zhang, J. Y.; Xiang, B. Synthesis and Enhanced Electrochemical Catalytic Performance of Monolayer $\mathrm{WS}_{2(1-x)} \mathrm{Se}_{2 x}$ with a Tunable Band Gap. Adv. Mater. 2015, 27, 4732-4738. 
6. Berry, J.; Ristić, S.; Zhou, S.; Park, J.; Srolovitz, D. J. The MoSeS Dynamic Omnigami Paradigm for Smart Shape and Composition Programmable 2D Materials. Nature Commun. 2019, 10, 5210.

7. Defo, R. K.; Fang, S.; Shirodkar, S. N.; Tritsaris, G. A.; Dimoulas, A.; Kaxiras, E. Strain dependence of band gaps and exciton energies in pure and mixed transition-metal dichalcogenides. Phys. Rev. B 2016, 94, 155310.

8. Kataria, S.; Wagner, S.; Cusati, T.; Fortunelli, A.; Iannaccone, G.; Pandey, H.; Fiori, G.; Lemme, M. C. Growth-Induced Strain in Chemical Vapor Deposited Monolayer $\mathrm{MoS}_{2}$ : Experimental and Theoretical Investigation. Adv. Mater. Interfaces 2017, 4, 1700031.

9. Kumar, N.; Najmaei, S.; Cui, Q.; Ceballos, F.; Ajayan, P. M.; Lou, J.; Zhao, H. Second Harmonic Microscopy of Monolayer $\mathrm{MoS}_{2}$. Phys. Rev. B 2013, 87, 161403.

10. Li, Y.; Rao, Y.; Mak, K. F.; You, Y.; Wang, S.; Dean, C. R.; Heinz, T. F. Probing Symmetry Properties of Few-Layer $\mathrm{MoS}_{2}$ and h-BN by Optical Second-Harmonic Generation. Nano Lett. 2013, 13, 3329-3333.

11. Malard, L. M.; Alencar, T. V.; Barboza, A. P. M.; Mak, K. F.; de Paula, A. M. Observation of Intense Second Harmonic Generation from $\mathrm{MoS}_{2}$ Atomic Crystals. Phys. Rev. B 2013, 87, 201401.

12. Lu, A. Y.; Zhu, H. Y.; Xiao, J.; Chuu, C. P.; Han, Y. M.; Chiu, M. H.; Cheng, C. C.; Yang, C. W.; Wei, K. H.; Yang, Y. M.; Wang, Y.; Sokaras, D.; Nordlund, D.; Yang, P. D.; Muller, D. A.; Chou, M. Y.; Zhang, X.; Li, L. J. Janus Monolayers of Transition Metal Dichalcogenides. Nat. Nanotechnol. 2017, 12, 744-749.

13. Ceballos, F.; Cui, Q.; Bellus, M. Z.; Zhao, H. Exciton Formation in Monolayer Transition Metal Dichalcogenides. Nanoscale 2016, 8, 11681-11688. 